\title{
Estimation of Transverse Mixing Coefficient in Straight and Meandering Streams
}

Mohammad Aghababaei $^{\mathrm{a}}$, Amir Etemad-Shahidi ${ }^{\mathrm{b}, \mathrm{c}_{*}}$; Ebrahim Jabbari ${ }^{\mathrm{d}}$ and Milad Taghipour ${ }^{\mathrm{e}}$

${ }^{a}$ M.Sc., School of Civil Engineering, Iran University of Science and Technology,

Narmak, Tehran, Iran, E-mail: moha.aghababaei@gmail.com

${ }^{\mathrm{b}} \mathrm{PhD}$, Griffith School of Engineering, Griffith University, Gold Coast, 4222, Australia

Tel: +61 7 55529267, Fax: +61 755528065

E-mail: a.etemadshahidi@griffith.edu.au,Corresponding author

${ }^{\mathrm{b}} \mathrm{PhD}$, School of Engineering, Edith Cowan University, Joondalup, 6027, Australia

${ }^{\mathrm{d}} \mathrm{PhD}$, School of Civil Engineering, Iran University of Science and Technology,

Narmak, Tehran, Iran, E-mail: jabbari@iust.ac.ir

${ }^{\mathrm{e}} \mathrm{PhD}$ Student, Civil, Geological and Mining Engineering, Poly technique Montréal, C.P.6079, Station Centre-ville, Montréal, Québec H3C 3A7, Canada

E-mail: milad.taghipour@polymtl.ca 


\begin{abstract}
Transverse mixing coefficient (TMC) is one of the key factors in the modelling of lateral dispersion of pollutants. Several researchers have attempted to estimate this coefficient using various models. However, robust equations that can accurately estimate lateral mixing in both straight and meandering streams are still required. In this study, novel formulae were developed using the hydraulic and geometric parameters of rivers. The multiple linear regression (MLR), genetic programming based symbolic regression (GPSR) and dimensionless parameters were used for this purpose. Two extensive data sets including data from straight channels/streams and meandering ones were employed to develop the formulae. The main advantage of the developed formula for meandering streams is proper consideration of the effects of aspect ratio, friction, and sinuosity. The formulae performances were then compared quantitatively with those of existing ones using accuracy metrics such as RMSE (Root Mean Square Error). The results illustrated that the proposed formulae outperform others in terms of accuracy and can be used for estimating TMC in straight and meandering streams. In addition, the comparison of MLR and GPSR models showed that the latter is marginally more accurate than MLR specially in meandering streams. However, the MLR models presented a more justifiable relationship between the TMC and governing dimensionless parameters. The main advantages of the presented formulae are that they are more accurate than previous models, can be used in both meandering and straight streams; and can be easily implemented in numerical models to estimate the pollutant concentration and mixing length.
\end{abstract}

Key words: Transverse mixing; Genetic programming, based symbolic regression; Straight streams; Meandering streams; Sinuosity

\title{
1. Introduction
}


Surface water quality modeling is a challenging issue for scientists and engineers. Discharge of pollutants into receiving waters deteriorates the quality of waters as they are transported by advection and diffusion processes in the water body. Understanding the mixing mechanism of pollutants in natural streams is a necessary step for determination of water quality and pollutants control in rivers (Sharma and Ahmad 2014). The mixing of pollutants in rivers is known to occur in three different directions; longitudinal, vertical and transverse or lateral (Rutherford1994). Vertical mixing usually occurs quickly in rather shallow water depth near discharging points in natural rivers and hence can be ignored in modeling of tracer clouds compared with lateral and longitudinal mixing processes (Jeon et al. 2007). Given the longitudinal mixing being less important when the load source is steady (Rutherford, 1994), transverse mixing becomes critical in estimating the impact of pollutants under steady state conditions (Boxall et al. 2003). In fact, transverse mixing has been distinguished to play a more important role in water quality management; especially in a case of point source discharges or tributary inflows (Rutherford 1994).

According to the principle of conservation of mass, distribution of tracer concentration can be presented in a two-dimensional model (Rutherford 1994):

$$
\frac{\partial}{\partial x}\left(D U_{x} C\right)+\frac{\partial}{\partial z}\left(D U_{z} C\right)=\frac{\partial}{\partial z}\left(D E_{z} \frac{\partial C}{\partial z}\right)
$$

Where $C$ is the depth-averaged tracer concentration $\left(\mathrm{kg} / \mathrm{m}^{3}\right) ; x$ and $\mathrm{z}$ are longitudinal and transverse directions, respectively; $D$ is the local depth $(m)$; $U_{x}$ and $U_{z}$ are velocities in the $x$ and $z$ directions $(\mathrm{m} / \mathrm{s})$, respectively and $E_{z}$ is the TMC $\left(\mathrm{m}^{2} / \mathrm{s}\right)$. By assuming a uniform flow and $U_{z}=0$, eq.1 is simplified to: 


$$
U_{x} \frac{\partial C}{\partial x}=E_{z} \frac{\partial^{2} C}{\partial z^{2}}
$$

The simplifying assumptions used to derive eq.2 do not necessarily occur in natural streams, and it may not represent all cases. However, this equation provides a useful basis for an initial study of the transverse mixing process (Rutherford 1994).

Generally, there are two main approaches utilized in the literature for estimating the TMC: theoretical and empirical. The theoretical approach deals with the concept of shear flow while the empirical one uses channels hydraulic and geometric properties in order to establish a relationship for $E_{z}$ (Baek and Seo 2013). Many investigators attempted to estimate the TMC by either the theoretical approach (e.g. Baek and Seo 2013) or the empirical approach (e.g. Chau 2000; Holley and Abraham 1973; Krishnappan and Lau 1977; Yotsukura and Sayre 1976). Several laboratory-based studies tried to estimate this coefficient through various types of laboratory channels from straight (e.g. Azamathulla and Ahmad 2012; Chau 2000) and meandering ones (e.g. Baek and Seo 2013; Yotsukura and Sayre 1976) while use of field data sets may not be straightforward due to the scarcity of field data (Beltaos 1979). Obtaining a general formula for estimating the TMC provides an opportunity to characterize the mixing mechanism while lowering the needs for time-consuming tracer studies that are costly (EtemadShahidi and Taghipour 2012). As a result, many researchers have focused on providing formulae for estimation of this coefficient (e.g. Azamathulla and Ahmad 2012; Baek and Seo 2013; Chau 2000; Elder 1959; Fischer et al. 1979; Jeon et al. 2007; Yotsukura and Sayre 1976). Although, the previous studies have provided invaluable knowledge about the role of different parameters, some issues still remain unsolved. For example, Webel and Schatzmann (1984) reported that dimensionless TMC is reduced by decreasing the friction term, while Chau (2000) observed no 
change in the TMC under various bottom roughness conditions. Recently, Pilechi et al. (2016), using a combination of field data and numerical modeling, noticed the importance of other parameters such as sinuosity and bed irregularity on the TMC. Therefore, more detailed investigations on the role of influencing parameters in transverse mixing seem to be required; and a more reliable predictive tool would be beneficial.

The main aim of this study is to develop a robust model for the estimation of transverse mixing coefficients (TMC) in straight and meandering rivers, by applying the genetic programming based symbolic regression (GPSR) and multiple linear regression (MLR). GPSR is one of the stochastic techniques that uses a set of functions (as possible solutions) to find the best solution for particular problem. The remarkable advantage of GPSR is that functional forms of solution do not have to be pre-assumed. In other words, GPSR tries to find the most accurate model without any constraints originated from specific functional form of solution. As discussed by Can and Heavy (2012), GPSR is superior in terms of accuracy of prediction than some other soft computing methods such as artificial neural network (ANN). In addition, it is more compact and utilizes fewer parameters. GPSR has been successfully tested in some engineering problems such as estimation of heat transfer correlations (Cai et al. 2005). MLR is one of the regression techniques that tries to create significant linear relationship between several independent and dependent variables (Montgomery et al. 2012). The main advantage of this method is to provide mathematically transparent relationship between variables. The technique has been frequently applied to many water engineering cases such as prediction of the load from total bed material in rivers (Sinnakaudan et al. 2006). To the best of the author's knowledge, these techniques have not been used in the estimation of TMCs so far.

\section{Background}


Previous investigations have shown that TMC is related to various parameters such as flow depth, channel width, mean velocity, friction factor, shear velocity, radius of curvature, horizontal stream curvature or sinuosity (e.g. Beltaos 1979; Fischer 1973; Krishnappan and Lau 1977; Lee and Seo 2013). Previous attempts have mostly been devoted to introducing a formula for estimating $E_{z}$ from easily measurable parameters. Fischer and Park (1967) proposed an experimental equation using field measurements in a seemingly straight irrigation canal with a loose sand bed:

$E_{z}=0.23 D U_{*}$

Where $U_{*}$ is the bed shear velocity $(\mathrm{m} / \mathrm{s})$. The channel side was reported to be straight generally. However, some parts were meandered (approximately $120 \mathrm{~m}$ of the channel). They concluded that the existence of curvature in these parts is responsible for the increase in transverse mixing. By considering the friction term and ratio of depth-to-curvature radius in small scale experiments, Fischer (1969) offered a new formula for TMC in a uniformly curving open channel:

$$
E_{z}=0.25 \frac{U^{2} D^{3}}{\kappa^{5} R_{c}{ }^{2} U_{*}}
$$

In which $U$ is the cross-sectional average velocity $(\mathrm{m} / \mathrm{s}), R_{C}$ is the radius of curvature of the channel center line $(\mathrm{m})$ and $\kappa$ is the Von Karman constant $(\kappa \approx 0.41)$. It can be worthy of notice that if $R_{C}$ tends to infinity, i.e. straight channel, then $E_{z}$ tends to become zero. Hence, there must be a limit for the applicability of Eq.4. Fischer (1969) illustrated that dimensionless form of $E_{z}$ $\left(E_{z} / D U_{*}\right)$ is largely affected by channel curvature. However, Rutherford (1994) stated that this 
equation can underestimate transverse mixing in natural streams due to the difference in flow structure. Fischer et al. (1979) determined the range of TMC as:

$\frac{E_{z}}{D U_{*}}=0.3-0.9$

When the sidewall of channel was moderately irregular and the stream was slowly meandering.

TMC was also measured in Missouri River by Yotsukura et al. (1970) and using the mometum method they reported that:

$E_{z}=0.70 D U_{*}$

In the moment method, TMC was obtained through eq. 6-b, proposed by Sayre and Chang (1969) as:

$E_{z}=\frac{U}{2} \frac{d \sigma_{z}^{2}}{d x}$

In which $x$ is distance downstream from the point of injection of the tracer, and $\sigma_{z}{ }^{2}$ is the variance of the transverse distribution of the tracer. $E_{z}$ was then obtained by eq.6-b; and eventually using measured mean depth and mean shear velocity of the stream, eq.6-a was developed. Yotsukura and Sayre (1976) proposed a formula by using the width of channel instead of mean depth, and suggested the following formula:

$$
\frac{E_{z}}{D U_{*}}=\alpha\left(\frac{U}{U_{*}}\right)^{2}\left(\frac{B}{R_{c}}\right)^{2}
$$

Where $B$ is the width of flow, and $\alpha$ is the proportional constant that was reported 3.4 by Yotsukura and Sayre (1976). This constant value was obtained for $12 \mathrm{~km}$ of natural streams with 
bends of $90^{\circ}$ and $180^{\circ}$. By implementing several experiments, including different bottom roughness conditions; Chau (2000) concluded that the TMC remains almost unaltered under these conditions. According to the experimental results, the following formula was proposed:

$E_{z}=0.18 D U_{*}$

By considering the aspect ratio $(B / D)$, some researchers explored other formulae for estimating the TMC (e.g. Deng et al. 2001; Gharbi et al. 1998). Deng et al. (2001) developed an equation based on the data measured in 11 large rivers in the United States as:

$$
\frac{E_{z}}{D U_{*}}=0.145+\left(\frac{1}{3520}\right)\left(\frac{U}{U_{*}}\right)\left(\frac{B}{D}\right)^{1.38}
$$

The studied cases consisted of both shallow and wide, and deep and narrow rivers. In addition to friction term $\left(U / U_{*}\right)$ and aspect ratio $(B / D)$, Jeon et al. (2007) considered the effect of sinuosity on the TMC in natural meandering streams using the least square method and suggested the following formula:

$$
\frac{E_{z}}{D U_{*}}=0.0291\left(\frac{U}{U_{*}}\right)^{0.463}\left(\frac{B}{D}\right)^{0.299}\left(S_{n}\right)^{0.733}
$$

In which $S_{n}$ is the sinuosity defined as the ratio between the thalweg length and the down-valley distance with a minimum value of 1 .

Azamathulla and Ahmad (2012), applying gene-expression programming (GEP) to laboratory data of straight rectangular channels, presented the following equation: 
$\frac{E_{z}}{D U_{*}}=\frac{\log (\log (\hat{U}+\hat{B}))}{\sin (-5.84 \hat{B})-\hat{U}+2.765}+\frac{\hat{U}^{\sin (8.22 \hat{B})}}{\hat{B}^{2}-645.42}+\frac{0.35 \hat{B}}{\operatorname{Ln}(\hat{U})+2 \hat{B}}$

Where $\hat{B}=\frac{B}{D}$ and $\hat{U}=\frac{U}{U_{*}}$.

Although, eq.11 has an acceptable accuracy in comparison with other equations in straight streams (see section 5.1), it does not provide a transparent and justifiable functional form for transverse mixing rate. Hence, the role of governing parameters on $E_{Z} / D U_{*}$ is not transparent.

Baek and Seo (2013) theoretically introduced a formula to predict the TMC in river bends:

$$
\frac{E_{z}}{D U_{*}}=(77.88 P)^{2}\left(1-\exp \left(-\frac{1}{77.88 P}\right)\right)^{2}
$$

In which $P$ can be obtained using following formula:

$$
P=\left(\frac{U}{U_{*}}\right)\left(\frac{D}{R_{c}}\right)
$$

The effects of other parameters on the TMC have been studied as well. Tabatabaei et al. (2013) experimentally investigated the effect of bed formations in terms of dunes and vegetation. Their experiments indicated that the existence of dunes and vegetation on the channel wall enhances mixing in lateral direction. Heard et al. (2001) studied the transverse mixing in simple streams (which were approximately straight with moderate variation in width and depth) and complex streams (which had depth variations from 5 to $50 \mathrm{~cm}$ and included large debris, sharp turns, pools and constrictions) and found that the mixing occurs more rapidly in complex streams than in straight and simple ones. 


\section{Material and Method}

\section{1. $\quad$ Multiple Linear Regression (MLR)}

Multiple linear regression (MLR) fits an observed dependent data set using a linear combination of independent variables. If there are one dependent variable such as $y$ and several independent variables such as $x_{1}, x_{2}, \ldots$ and $x_{k}$ ( $k$ is the number of independent variables), the general form of multiple linear regression is as:

$y=\beta_{0}+\beta_{1} x_{1}+\beta_{2} x_{2}+\ldots+\beta_{k} x_{k}$

In which the parameters $\beta_{k}(k=1,2, \ldots, k)$ are called the regression coefficient.

\subsection{Genetic Programming (GP)}

GP is one of the artificial intelligence methods that uses a random iterative searching process to reach an appropriate relationship between input and output tree-structure base. The significant advantage of this structure is the application of a wide range of functions and variables in a malleable tree-structure base, which makes GP a powerful tool. The first expression of GP was given by Cramer (1985) and later developed by Koza (1992). GP has been developed based on Darwin's evolution theory (Darwin 1859). Similar to this process, in GP the best model is selected based on fitness function. In fact, among different models, the one that has most adaptability with fitness function is considered as the best model by GP (Azamathulla and Ghani 2011). GP has been used to solve complex water engineering problems recently (Dorado et al. 2003; Giustolisi 2004; Kalra and Deo 2007; Ustoorikar and Deo 2008; Azamathulla and Ghani 2011; Fallah-Mehdipour et al. 2014).

\subsection{Symbolic Regression (SR) and Genetic Programing based Symbolic Regression (GPSR)}


SR is one of the various kinds of regression analysis that can find the model that best fits a given dataset, both in terms of accuracy and simplicity. This is performed by searching the space of mathematical expressions by SR. The traditional regression usually presents models that are limited to linear or quadratic form. This shackle sometimes leads to less precise models; while, SR attempts to create a relationship between independent variables (input) and dependent one (output) without any predefined assumptions about the model's structure that can be led to best fitting especially in more complex problems(Gandomi, et al. 2015). As an earliest application of GP, symbolic regression has been used widely (e.g. Koza and Poli 2005; Schmidt and Lipson 2009).

GPSR is a mixing of evolutionary and regression methods to construct an unknown function with dataset, which probably are encountered with over-fitting (Vladislavleva et al. 2009). In this method, first of all, a random population of solutions is created to the function identification problem. The populations are generated in the form of a tree structure which originates from a root node and includes operator ("function set") and operand ("terminal”) nodes (Gandomi, et al. 2015). The terminals are the nodes without any branches that consist of variations and constants, while function set are the nodes with branches that are consisted of calculated operators and mathematical functions (Walker 2001). Generally, the features and steps of this method are similar to those of GP. One of the main reasons of using GPSR in this research is that it investigates and optimizes the appropriate form of a linear or nonlinear data-fitting function without any pre assumption function form.

\subsection{Data Set}

Two main data sets used in this study consist of 181 measurements in straight channels/streams (including 9 field studies and 172 flume experiments) and 49 measurements in meandering ones 
(including 29 field cases and 20 flume experiments) with a wide range of parameters. These data were extracted from the literature (i.e. Beltaos 1979; Holley and Abraham 1973; Jeon et al. 2007; Krishnappan and Lau 1977; Rutherford 1994; Baek and Seo 2008; Yotsukura et al. 1970, Lee and Seo 2013). All of the data sets contain hydraulic and geometric parameters including flow width, depth, mean velocity, shear velocity and Froude number for straight and meandering cases. In addition, sinuosity data has been collected for meandering streams. It is worth mentioning that due to the lack of measured $R_{c} S_{n}$ has been used to demonstrate horizontal irregularities of streams. Approximately $85 \%$ of the $E_{z} / D U_{*}$ values appear to be between 0.1 and 0.2 in straight channels/streams. In addition, nearly $81 \%$ of the $E_{z} / D U_{*}$ values vary between 0.2 and 0.9 in meandering streams presenting a good agreement with that of the range reported in Rutherford (1994) which is $.0 .15<E_{z} / D U_{*}<0.30$ in straight channels and $0.30<E_{z} / D U_{*}<0.90$ in meandering channels. The high values of $E_{z} / D U_{*}$ in these cases is mostly due to the existence of secondary flow and geometric irregularity (Rutherford 1994). As an example, Baek and Seo (2011) derived a predictive modeling for curved channels by considering the effect of secondary flow on transverse mixing process and found a higher TMC in curved channels. Froude number in straight and meandering cases varied from 0.02 to 0.97 , indicating a prevalent subcritical condition. Table 1 provides more statistics of the data used in this study. As seen, the variation of $B / D$ in streams indicates that the studied cases varied from narrow streams $(B / D<10)$ to very wide ones $(B / D>100)$. In addition, the range of $S_{n}$ in meandering streams is between 1.03 and 3.33, implying various meandering conditions ranging from nearly straight to sharply meandering ones.

\section{Models Development}

\subsection{Determination of Effective Parameters}


As mentioned before, there are various parameters that influence the TMC. Hence, defining governing parameters and employing them in the model development step is significantly important for comprehensive results. The following effective parameters reported in the literature can be used as the input and output parameters:

$$
E_{z}=f_{1}\left(B, D, U, U_{*}, \rho, \mu, S_{f}, g, S_{n}\right)
$$

Where $\rho$ is the fluid density; $\mu$ is the fluid viscosity; $S_{f}$ is the bed shape factor; $g$ is the gravity and $S_{n}$ is the sinuosity. $S_{f}$ and $S_{n}$ indicate the irregularity in vertical and horizontal directions, respectively (Etemad-Shahidi and Taghipour 2012). $R_{c}$ can be approximately replaced by $S_{n}$ as it reflects curvature of the streams. In fact, using $S_{n}$ is preferred since it is the ratio of thalweg length to the direct distance between two points in the channel which is a constant value and in contrast with $R_{c}$, it does not need to be measured for each curvature of the channel (Lau and Krishnappan 1981). Based on previous researches (e.g. Jeon et al. 2007; Fischer 1973;

Azamathulla and Ahmad 2012) $U *$ is also considered as a governing parameter in this study. This parameter is calculated as $\sqrt{g D S_{f}}$ in the steady uniform open channel flow. Due to turbulence, the transverse mixing is controlled by bed shear velocity in transverse direction. Therefore, this is a significant parameter that should be considered in the modeling of TMC (Azamathulla and Ahmad 2012). Based on previous studies and using dimensional analysis, eq.14 can be written in the dimensionless form (Fischer et al. 1979; Jeon et al. 2007):

$$
\frac{E_{z}}{D U_{*}}=f_{2}\left(\frac{B}{D}, \frac{U}{U_{*}}, \rho \frac{D U}{\mu}, S_{f}, \frac{U}{\sqrt{g D}}, S_{n}\right)
$$

In which $\rho D U / \mu$ is the Reynolds number. All laboratory data that have been used in this study were obtained from uniform rectangular channels, so hydraulic depth was equal to the local 
depth. Therefore, the depth of channel is used in calculation of Froude number $(U / \sqrt{g D})$.

According to Besio et al. (2012), increasing in Froude number results in increasing of TMC in subcritical flows. In addition, recently, Sattar and Gharebaghi (2015) showed the importance of $\mathrm{Fr}$ in the prediction of longitudinal dispersion coefficient. The data sets of this study are mostly from turbulent flows. Therefore, the effect of the Reynolds number is negligible and can be ignored (Chau 2000). Since friction term $\left(U / U_{*}\right)$ may be able to reflect the irregularities of bed shape, it can be used instead of $S_{f}$ (Jeon et al. 2007).

It needs to be mentioned that the correlation between various dimensionless forms of TMC $\left(E_{z} / D U, E_{z} / B U, E_{z} / D U *\right.$ and $\left.E_{z} / B U_{*}\right)$, and the input parameters including $B / D, U / U_{*}, F_{r}$, showed that $E_{z} / D U_{*}$ is the best representative of the dimensionless TMC. Previous researchers have also introduced dimensionless form of TMC (e.g Deng et al. 2001; Jeon et al. 2007; Yotsukura and Sayre 1976). Hence, eq.15 can be simplified as:

$$
\frac{E_{z}}{D U_{*}}=f_{3}\left(\frac{B}{D}, \frac{U}{U_{*}}, F_{r}, S_{n}\right)
$$

According to previous investigations and by assuming $f_{3}$ as a power function, the general expression of the TMC can be written as:

$$
\frac{E_{z}}{D U_{*}}=a\left(\frac{B}{D}\right)^{b}\left(\frac{U}{U_{*}}\right)^{c}\left(F_{r}\right)^{d}\left(S_{n}\right)^{e}
$$

Where $a, b, c, d$ and $e$ are constants that can have different values in different conditions. eq.17 is a general expression of the influencing parameters for meandering channels/streams. In straight cases ( sinuosity $=1)$ eq. 17 becomes: 


$$
\frac{E_{z}}{D U_{*}}=a\left(\frac{B}{D}\right)^{b}\left(\frac{U}{U_{*}}\right)^{c}\left(F_{r}\right)^{d}
$$

The test-and-train technique is commonly used in developing formulae by data sets (e.g.

Kazeminezhad et al. 2010; Hadadpour et al. 2013). The statistical properties of 122 (out of 181) training data points of straight channels/streams are shown in Table 1. Since the output of this model is a linear function, logarithm of parameters needs to be applied. As a result, eq.18 was converted to:

$\log \left(\frac{E_{z}}{D U_{*}}\right)=\log a+b \log \left(\frac{B}{D}\right)+c \log \left(\frac{U}{U_{*}}\right)+d \log \left(F_{r}\right)$

\section{2. $\quad$ MLR Model}

Using MLR analysis, values of $a, b, c, d$ were obtained and the following formula was achieved for TMC in straight cases as:

$$
\frac{E_{z}}{D U_{*}}=0.159\left(\frac{B}{D}\right)^{0.126}\left(\frac{U}{U_{*}}\right)^{-0.148}
$$

It should be mentioned the regression analysis showed that $\mathrm{Fr}$ has no significance role on transverse mixing rate which is in line with the finding by Webel and Schatzmann (1984).

Having obtained eq.20 for straight streams, sinuosity $\left(S_{n}\right)$ still needs to be accounted for in nonstraight streams as it has been recognized as an important parameter in meandering streams. In this regard, Boxall et al. (2003) studied the effect of this parameter on lateral mixing and reported that there is direct relationship between the TMC and the sinuosity. Moreover, numerical studies of Demuren and Rodi (1986) showed that the effect of channel curvature on the mixing phenomenon is important in the narrow channels with the smooth bed. It can be 
inferred through their investigation that the sinuosity effect on the TMC depends on the aspect ratio and friction terms. Jeon et al. (2007) took a step further and used a power function to include the effect of sinuosity on TMC. Based on their work and physical justification, it was decided to consider sinuosity effect as a power function of $B / D$ and $U / U *$ for meandering streams. Different functional forms were tested by using MLR technique which led to following for estimating of $E_{z}$ in meandering streams:

$\frac{E_{z}}{D U_{*}}=0.159\left(\frac{B}{D}\right)^{0.126}\left(\frac{U}{U_{*}}\right)^{-0.148}\left[1+\left(0.501\left(\frac{U}{U_{*}}\right)^{0.447}\left(S_{n}-1\right)^{0.275}\right)\right]$

\subsection{GPSR Model}

The main issue for building GPSR model is determining of the operator and operand. Selection of appropriate operands and operators was based on the previous formulae, so that those ones that were most frequently appeared in existing models were chosen. Accordingly, addition, subtraction, multiplication and power operations have been considered as functions sets and $B / D$, $U / U_{*}, F_{r}, S_{n}$ and constant number as terminals. The testing and training data were the same as those used in the MLR model. Generation of different combinations of formulae for meandering streams were examined and finally; the following is obtained as there was no improvement in the value of fitness function:

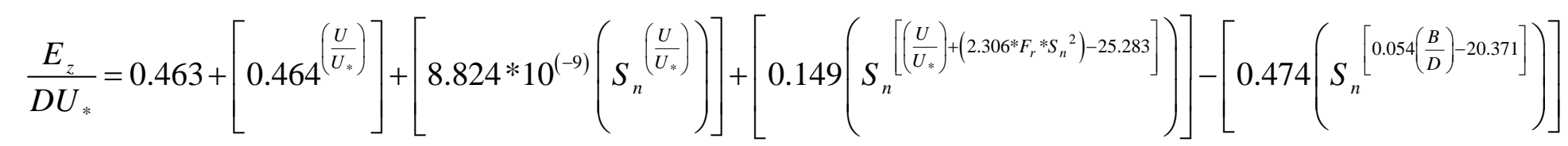

The simplified version of eq.22 applicable for straight streams can be achieved by assuming $S n=1$ as: 


$$
\frac{E_{z}}{D U_{*}}=0.138+\left[0.464^{\left(\frac{U}{U_{*}}\right)}\right]
$$

\section{Discussion}

\subsection{Evaluation of the Proposed Models}

In order to compare the performances of existing and developed formulae, accuracy metrics such as the discrepancy ratio $(D R)$; mean of the absolute error $(M E)$; and root mean square (RMSE) were used for the evaluation and comparison of the developed formula with the other existing models:

$D R_{i}=\log \frac{E_{z_{p}}}{E_{z_{m}}}$

$M E=\frac{1}{N} \sum_{i=1}^{N}\left|D R_{i}\right|$

$R M S E=\sqrt{\frac{1}{N} \sum_{i=1}^{N}\left(D R_{i}\right)^{2}}$

Where $E_{z_{p}}$ and $E_{z_{m}}$ are the predicted and measured TMCs, respectively; and $N$ is the total number of data points. $D R$ parameter shows the discrepancy between the predicted and measured values. $D R$ equal to zero indicates the exact match of predicted and measured values. Otherwise an overestimation $(D R>0)$ or underestimation $(D R<0)$ occur. In addition, percentage of $D R$ values that fall between -0.3 and 0.3 is defined as the accuracy of a model (Zeng and Huai 2014). $M E$ and RMSE are also statistical parameters for the determination of model performance. The closer the values of these parameters to zero, the more accurate the model will be. Table 2 presents the calculated accuracy metrics of the developed formulae including MLR and GPSR 
models (Eqs.20 and 23 respectively) and previous ones for both testing and all data sets of straight streams. It can be concluded from Table 2 that the performance of Eq.20 is almost the same for both testing and all data. In addition, same as MLR formula, Eq.23 has better accuracy metrics than previous formulae. Table 2 also shows that model of Chua (2000) outperforms other existing models in estimating of TMC in straight streams. Therefore, it was decided to use the data sets used in developing the model of Chua (2000) for a fairer comparison of the models. It can be inferred from Table 2 that the performances of eq. 20 and Chua's one are nearly the same for this selected data base. Values of $D R_{i}$ do not show a symmetric distribution (Table 2) even though the percentage of the predictions with the $D R$ values between -0.3 to 0.3 are about $98 \%$; of which $83 \%$ are in the range of 0 to 0.3 ; and $15 \%$ are in the range of -0.3 to 0 . In summary, the proposed formulae (Eqs. 20 and 23) outperform other ones (considering DR, ME and RMSE) and also their predictions are symmetrically distributed around zero for both testing and all data. Investigating $D R$ variations versus input parameters is another method to evaluate the formula performance. If the errors of the model are independent of input parameter values, the performance of a model will be considered better than other ones (e.g. Jafari and Etemad-Shahidi 2012). Fig. 1 shows that the $D R$ values are almost independent of the input parameters including $B / D$ and $U / U_{*}$ when using MLR and GPSR models, respectively. The low values of determination coefficients and slopes of the best fit lines imply that there is no systematic error when using these formulae. While, it can be concluded that the $D R$ values of the other models are more dependent on the input parameters, including $B / D$ and $U / U_{*}$.

The performance of MLR and GPSR models of meandering streams (eqs. 21 and 22, respectively) were compared with that of Jeon et al. (2007) which includes the role of sinuosity. As seen in Table 2, about $33 \%$ of $D R$ values of Jeon et al. (2007) formula were lower than -0.3 
showing underestimated predictions while the corresponding value of eqs. 21 and 22 are about 22\% and 6\%, respectively. Both ME and RMSE of Jeon et al. (2007) formula are higher than those of eqs. 21 and 22. This could also be due to the limited data set used in the former study. In addition, approximately $75.5 \%$ of the $D R$ values of eq. 21 and $82 \%$ of those of eq.22 fall between -0.3 and 0.3 symmetrically. Besides, by applying Jeon et al. (2007)’ data and comparing the performance of new proposed formula with theirs (Table 2), it can be concluded that eqs. 21 and 22 estimate TMC in meandering streams more accurately than Jeon et al. (2007) formula. In brief, it can be concluded that the developed formulae are skillful in both straight and meandering streams. However, GPSR model (Eq.22) performs better than MLR model (Eq.21) and Jeon et al. (2007) in estimating TMC in meandering streams. Scatter diagram of the predicted $E_{z} / D U_{*}$ versus measured ones is shown in Fig. 2. As seen, predictions of the GPSR model are closer to the best fit line than those of MLR and Jeon et al. (2007); suggesting the more skillfulness of eq.22 in comparison with others.

\subsection{Parametric Analysis and Application}

The effects of $B / D$ and $U / U *$ on the TMC in straight streams have been investigated using previous formulae and the developed MLR and GPSR models in Fig. 3a. As shown, MLR model implies that by increasing the aspect ratio, the TMC increases. The lower $B / D$ values which correspond to narrower and deeper cases, result in lower TMCs. This finding is in line with the existing knowledge found by Seo et al. (2006) where the TMCs in narrow and deep parts of Han river in Korea were observed to be lower than those of the shallow and wide parts. Jeon et al. (2007) model also shows the same behavior, as $E z / D U *$ increases by increasing $B / D$. In addition, according to Fig. 3a, there is not a clear relationship between $B / D$ and $E z / D U *$ for model of 
Azamathulla and Ahmad (2012). Therefore, this model may suffer from the lack of physical justification.

Variation of $E z / D U *$ versus $U / U * \mathrm{~h}$ is also shown in Fig. 3b. According to this figure, it can be inferred from MLR and GPSR models that the TMC is inversely proportional to the friction term in straight streams. However, these results are not in agreement with those by Jeon et al. (2007). It seems, the results of this study about the relationship between $U / U *$ and $E z / D U *$ is more compliant with the physics of transverse mixing phenomena. Friction term is related to Darcy Weisbach friction factor through the following formula (Chau 2000):

$f=8\left(\frac{U_{*}}{U}\right)^{2}$

In which, $f$ is the Darcy Weisbach friction factor. eq.27 shows that Darcy Weisbach friction factor increases by decreasing $U / U_{*}$. Based on eq.27, the TMC is directly related to this factor that reflects the role of bed friction and generated turbulence. It had been already reported by Bruno et al. (1990) that TMC in a rough bed is higher than that of a smooth bed. Another significant point that can be inferred from Fig. $3 \mathrm{~b}$ is independent relationship between $U / U *$ and $E z / D U_{*}$ at high values of $U / U_{*}$ (more than 20 ). As seen, $E z / D U *$ values become gradually uncorrelated with $U / U *$ for MLR, GPSR and Azamathulla and Ahmad (2012) models. It means that in very smooth channels, transverse mixing becomes independent of bottom friction. This could be due to the fact that in smooth channels, the bottom friction has no major role in generating turbulent mixing.

The developed formulae for meandering streams show that $E_{z}$ is directly related to the sinuosity. This is consistent with the previous findings of Seo et al. (2006). It is worth mentioning that 
MLR model (eq.21) shows that the effect of sinuosity depends on the friction term. This result is also in line with the findings of Demuren and Rodi (1986) who pointed out that the TMC is more influenced by $S_{n}$ as friction decrease (i.e. increase of $U / U_{*}$ ). The main advantage of developed models is that they offer a mathematical expression that can be used in both straight and meandering streams. By assuming $S_{n}=1$, eq. 21 and 22 are converted to eq.20 and 23 respectively; which are the formulae for straight streams.

\section{Conclusions}

In this study, multiple linear regression (MLR) and genetic programming based symbolic regression (GPSR) techniques were used to develop a robust model for estimation of TMC in meandering and straight streams. Scaling argument and data mining approach were used to develop unified formulae for prediction of (dimensionless) TMC as function of easily available hydraulic parameters such as aspect ratio, friction factor and sinuosity. Several accuracy metrics were used to assess the models' performance. Results showed the higher accuracy of the proposed models compared with the existing ones, both in laboratory and field experiments. The GPSR model was more accurate than MLR one in meandering-stream cases. However, the MLR model was simpler and more justifiable in terms of physical relationship between TMC and governing parameters. In straight-stream cases, both developed models showed the same level of accuracy. Given the better performance of GPSR one in meandering streams, it is finally concluded that GPSR can be considered as a more efficient tool for prediction of TMC. The results indicated that engineers can improve their designs using data driven approaches. The developed models can be used in inland water bodies, including straight and meandering rivers and channels. Developed formulae can be easily implemented in 2-D depth averaged or 3-D advection-dispersion numerical models to enhance their capacity, especially for the modelling of 
pollutant concentration in both near field and far field. 


\section{REFERENCES}

Azamathulla HM, Ahmad Z (2012) Gene-expression programming for transverse mixing coefficient. J Hydrol 434:142-148

Azamathulla HM, Ghani AA (2011) Genetic Programming for Predicting Longitudinal Dispersion Coefficients in Streams. Water Resour Manag 25:1537-1544

Baek KO, Seo IW (2008) Prediction of transverse dispersion coefficient using vertical profile of secondary flow in meandering channels. KSCE J Civ Eng 12:417-426

Baek KO, Seo IW (2011) Transverse dispersion caused by secondary flow in curved channels. J Hydraul Eng 137(10):1126-1134

Baek KO, Seo IW (2013) Empirical equation for transverse dispersion coefficient based on theoretical background in river bends. Environ Fluid Mech 13:465-477

Beltaos S, (1979) Transverse mixing in natural streams. Can J Civ Eng 6:575-591

Besio G, Stocchino A, Angiolani S, Brocchini M (2012) Transversal and longitudinal mixing in compound channels. Water Resour Res 48(12):1-15

Boxall JB, Guymer I, Marion A (2003) Transverse mixing in sinuous natural open channel flows. J Hydraul Res 41:153-165

Bruno MS, Muntisov M, Fischer HB (1990) Effect of buoyancy on transverse mixing in streams. J Hydraul Eng 116(12):1484-1494

Cai W, Sen M, Yang KT, Pacheco-Vega A (2005) Genetic-programming-based symbolic regression for heat transfer correlations of a compact heat exchanger. In ASME Heat Transfer Conf collocated with the ASME Pacific Rim Technical Conf and Exhibition on Integration and Packaging of MEMS, NEMS, and Electronic Systems 367-374

Can B, Heavey C (2012) A comparison of genetic programming and artificial neural networks in metamodeling of discrete-event simulation models. Comp and Oper Res 39(2):424-436

Chau KW (2000) Trasverse mixing coefficient measurements in an open rectangular channel. Adv Environ Res 4:287-294

Cramer NL (1985) A representation for the adaptive generation of simple sequential programs. In Proceedings of the First Intern Conf on Genetic Algorithms:183-187

Darwin C (1859) On the origins of species by means of natural selection. Murray, London 
Demuren AO, Rodi W (1986) Calculation of flow and pollutant dispersion in meandering channels. J Fluid Mech 172:63-92

Deng ZQ, Singh VP, Bengtsson L (2001) Longitudinal Dispersion Coefficient in Straight Rivers. J Hydraul Eng 127:919-927

Dorado J, Rabuñal JR, Pazos A, Rivero D, Santos A, Puertas J (2003) Prediction and modeling of the rainfall-runoff transformation of a typical urban basin using ANN and GP. Appl Arti l Intell 17:329-343

Elder JW, (1959) The dispersion of marked fluid in turbulent shear flow. J Fluid Mech 5:544560

Etemad-Shahidi A, Rohani MS (2012) Prediction of scour at abutments using piecewise regression. Proc ICE-Water Manag 167(2):79-87

Etemad-Shahidi A, Taghipour M (2012) Predicting Longitudinal Dispersion Coefficient in Natural Streams Using M5' Model Tree. J Hydraul Eng 138(6):542-554

Fallah-Mehdipour E, Haddad OB, Mariño MA (2014) Genetic programming in groundwater modeling. J Hydrol Eng 19(12):04014031

Fischer HB (1969) The effect of bends on dispersion in streams. Water Resour Res 5(2):496-506

Fischer HB (1973) Longitudinal dispersion and turbulent mixing in open-channel flow. Annu Rev Fluid Mech 5(1):59-78

Fischer HB, List EJ, Koh RCY, Imberger J, Brooks NH (1979) Mixing in Inland and Coastal Waters. Academic Press, New York

Fischer HB, Park M (1967) Transverse mixing in a sand-bed channel. Geol Surv Res 500:267272

Gandomi AH, Alavi AH, Ryan C (2015) Handbook of Genetic Programming Applications. Springer

Gharbi S, Verrette JL (1998) Relation between longitudinal and transversal mixing coefficients in natural streams. J Hydraul Res 36(1):43-54

Giustolisi O (2004) Using genetic programming to determine \{Chezy resistance coefficient in corrugated channels. J Hydroinf 6:157-173

Hadadpour S, Etemad-Shahidi A, Kamranzad B (2013) Wave energy forecasting using artificial neural networks in the Caspian Sea. Proc ICE-Maritime Eng 167(1):42-52 
Hair JF, Black B, Anderson R, Tatham R (1995) Multivariate Data Analysis: Text and Readings MacMillan, International Edition

Heard SB, Gienapp CB, Lemire JF, Heard KS (2001) Transverse mixing of transported material in simple and complex stream reaches. Hydrobiol 464:207-218

Holley ER, Abraham G (1973) Laboratory Studies On Transverse Mixing In Rivers. J Hydraul Res 11:219-253

Jafari E, Etemad-Shahidi A (2012) Derivation of a New Model for Prediction of Wave Overtopping at Rubble Mound Structures. J Waterw Port Coastal Ocean Eng 138:42-52

Jeon TM, Baek KO, Seo IW (2007) Development of an empirical equation for the transverse dispersion coefficient in natural streams. Environ Fluid Mech 7:317-329

Kalra R, Deo MC (2007) Genetic programming for retrieving missing information in wave records along the west coast of India. Applied Ocean Res 29:99-111

Kazeminezhad MH, Etemad-Shahidi A, Yeganeh-Bakhtiary A (2010) An alternative approach for investigation of the wave-induced scour around pipelines. J Hydroinf 12:51-65

Koza JR (1992) Genetic programming: on the programming of computers by means of natural selection. MIT press

Koza JR, Poli R (2005) Genetic programming. In: Burke EK, Kendall G, Search Methodologies:Introductory Tutorials in Optimization and Decision Support Techniques. 127164

Krishnappan BG, Lau YL (1977) Transverse Mixing in Meandering Channels With Varying Bottom Topography. J Hydraul Res 15:351-370

Kutner MH, Nachtsheim CJ, Neter J, Li W (1996) Applied linear statistical models. Springer, Chicago: Irwin

Lau YL, Krishnappan BG (1981) Modeling transverse mixing in natural streams. J Hydraul Div 107(2):209-226

Lee M, Seo W (2013) Spatially Variable Dispersion Coefficients in Meandering Channels. J Hydraul Eng 193(2):141-153

Pilechi A, Mohammadian A, Rennie CD, Zhu DZ (2016) Efficient Method for Coupling Field Data and Numerical Modeling for the Estimation of Transverse Mixing Coefficients in Meandering Rivers J Hydraul Eng 04016009

Rutherford JC (1994) River mixing. John Wiley and Sons Ltd 
Sattar A, Gharebaghi B (2015) Gene expression models for prediction of longitudinal dispersion coefficient in streams. J Hyrol 524:687-596

Sayre WW, Chang FM (1969) A laboratory investigation of open channel dispersion processes for dissolved, suspended, and floating dispersants. United States Geological Survey

Seo I, Baek KO, Jeon TM (2006) Analysis of transverse mixing in natural streams under slug tests. J Hydraul Res 44:350-362

Schmidt MD, H Lipson (2009) Solving iterated functions using genetic programming. In Proceedings of the 11th Annual Conf Companion on Genetic and Evolutionary Computation Conf Late Breaking Papers ACM:2149-2154

Sharma H, Ahmad Z (2014) Transverse mixing of pollutants in streams: a review Can J Civ Eng 41(5):472-482

Sinnakaudan SK, Ghani AA, Ahmad MS, Zakaria NA (2006) Multiple linear regression model for total bed material load prediction. J Hydraul Eng 132(5):521-528

Tabatabaei SH, Heidarpour M, Ghasemi M, Hoseinipour EZ (2013) Trasverse mixing coefficient on Dunes with Vegetation on a Channel Wall. World Environ Water Resour Congr ASCE:1903-1911

Ustoorikar K, Deo MC (2008) Filling up gaps in wave data with genetic programming. Marine Structures 21(2):177-195

Vladislavleva EJ, Smits GF, Den Hertog D (2009) Order of nonlinearity as a complexity measure for models generated by symbolic regression via pareto genetic programming. Evolutionary Computation IEEE Transactions on 13(2):333-349

Walker M (2001) Introduction to genetic programming. Tech Np, University of Montana

Webel G, Schatzmann M (1984) Transverse Mixing in Open Channel Flow. J Hydraul Eng 110(4):423-435

Yotsukura N, Fischer HB, Sayre WW (1970) Measurement of mixing characteristics of the Missouri River between Sioux City, Iowa, and Plattsmouth, Nebraska: US Geological Survey Water-Supply:1-29

Yotsukura N, Sayre WW (1976) Transverse mixing in natural channels. Water Resour Res 12(4):695-704

Zeng YH, Huai WX (2014) Estimation of longitudinal dispersion coefficient in rivers. J HydroEnvironment Res 8(1):2-8 
Table 1. Statistical properties of data sets used for developing the formula

\begin{tabular}{|c|c|c|c|c|c|c|c|c|c|}
\hline & \multicolumn{3}{|c|}{ Straight, training data } & \multicolumn{3}{|c|}{ Straight, testing data } & \multicolumn{3}{|c|}{ Meandering, all data } \\
\hline & Range & Average & $\begin{array}{l}\text { Standard } \\
\text { deviation }\end{array}$ & Range & Average & $\begin{array}{l}\text { Standard } \\
\text { deviation }\end{array}$ & Range & Average & $\begin{array}{l}\text { Standard } \\
\text { deviation }\end{array}$ \\
\hline$B / D$ & $1.67-132.6$ & 19.832 & 16.832 & $1.82-100$ & 18.015 & 16.605 & $2.5-287.5$ & 53.125 & 61.169 \\
\hline$U / U *$ & $4.48-23.11$ & 12.627 & 5.378 & $5.52-21.99$ & 13.502 & 4.831 & $\begin{array}{l}2.05- \\
28.57\end{array}$ & 13.085 & 6.083 \\
\hline$F_{r}$ & $0.09-0.97$ & 0.305 & 0.181 & $0.084-0.808$ & 0.320 & 0.193 & $0.06-0.58$ & 0.189 & 0.124 \\
\hline Sn & $1.0-1.0$ & 1.000 & 0.000 & $1.0-1.0$ & 1.000 & 0.000 & $1.03-3.33$ & 1.513 & 0.667 \\
\hline$E_{z} / D U_{*}$ & $0.055-0.946$ & $6 \quad 0.159$ & 0.084 & $0.081-0.30$ & 0.150 & 0.038 & $0.12-2.4$ & 0.543 & 0.395 \\
\hline
\end{tabular}


Table 2. Comparison of the performance of various formulae, straight and meandering streams

\begin{tabular}{|c|c|c|c|c|c|c|c|}
\hline Formula & $D R<-0.3$ & $-0.3<D R<0$ & $0<D R<0.3$ & $0.3<D R$ & Accuracy & $\mathrm{ME}$ & RMSE \\
\hline $\begin{array}{l}\text { Fischer and Park (1967), } \\
\text { (test, all, Chau } 2000 \text { data) }\end{array}$ & $(0.00,0.55,-)$ & $(5.09,7.18,-)$ & $(81.36,79.56,-)$ & $(13.56,12.71,-)$ & $(86.44,86.74,100)$ & $(0.20,0.21,0.12)$ & $(0.224,0.228,0.121)$ \\
\hline $\begin{array}{l}\text { Chau (2000), (test, all, } \\
\text { Chau } 2000 \text { data) }\end{array}$ & $(0.00,1.11,-)$ & $(15.25,14.92,-)$ & $(83.05,82.32,-)$ & $(1.70,1.66,-)$ & $(98.31,97.33,100)$ & $(0.12,0.12,0.03)$ & $(0.138,0.152,0.032)$ \\
\hline $\begin{array}{c}\text { Jeon et al. (2007), (test, all, } \\
\text { Chau } 2000 \text { data) }\end{array}$ & $(0.00,1.11,-)$ & $(8.48,14.92,-)$ & $(81.36,71.82,-)$ & $(10.17,12.16,-)$ & $(91.53,87.74,50)$ & $(0.15,0.17,0.29)$ & $(0.182,0.203,0.307)$ \\
\hline $\begin{array}{c}\text { Azamathulla and Ahmad } \\
\text { (2012), (test, all, Chau } 2000 \\
\text { data) }\end{array}$ & $(0.00,2.21,-)$ & $(37.29,35.91,-)$ & $(49.15,46.96,-)$ & $(13.56,14.92,-)$ & $(86.44,82.87,100)$ & $(0.15,0.17,0.10)$ & $(0.259,0.298,0.108)$ \\
\hline $\begin{array}{c}\text { MLR, (Eq.20), (test, all, } \\
\text { Chau } 2000 \text { data) }\end{array}$ & $(0.00,0.55,-)$ & $(44.07,42.54,-)$ & $(55.93,56.35,-)$ & $(0.00,0.55,-)$ & $(100.00,98.90,100)$ & $(0.08,0.09,0.02)$ & $(0.100,0.114,0.042)$ \\
\hline $\begin{array}{c}\text { GPSR, (Eq.23), (test, all, } \\
\text { Chau } 2000 \text { data) }\end{array}$ & $(1.70,1.66,-)$ & $(47.46,48.07,-)$ & $(50.85,49.72,-)$ & $(0.00,0.55,-)$ & $(98.31,97.79,100)$ & $(0.08,0.08,0.10)$ & $(0.105,0.123,0.108)$ \\
\hline $\begin{array}{c}\text { Jeon et al. (2007), (all, Jeon } \\
\text { et al. } 2007 \text { data) }\end{array}$ & $(32.65,-)$ & $(32.65,-)$ & $(34.69,-)$ & $(0.00,-)$ & $(67.35,95)$ & $(0.24,0.12)$ & $(0.315,0.191)$ \\
\hline $\begin{array}{l}\text { MLR (Eq.21), (all, Jeon et } \\
\text { al. } 2007 \text { data) }\end{array}$ & $(22.45,-)$ & $(42.86,-)$ & $(32.65,-)$ & $(2.04,-)$ & $(75.51,95)$ & $(0.22,0.12)$ & $(0.265,0.170)$ \\
\hline $\begin{array}{c}\text { GPSR (Eq.22), (all, Jeon } \\
\text { et al. } 2007 \text { data) }\end{array}$ & $(6.12,-)$ & $(38.78,-)$ & $(42.86,-)$ & $(12.25,-)$ & $(81.63,100)$ & $(0.15,0.10)$ & $(0.217,0.123)$ \\
\hline
\end{tabular}




\section{Figure Captions}

Fig. 1. Variation of $D R$ against $B / D$ and $U / U_{*}$ for (a) Jeon et al. (2007), (b) Azamathulla and Ahmad (2012), (c) MLR and (d) GPSR

Fig. 2. Comparison of observed and predicted $E_{z} / D U *$ using Jeon et al. (2007), MLR and GPSR models

Fig. 3. $E z / D U *$ versus (a) $B / D$ and (b) $U / U *$ for straight streams models $(B / D=19.067$ and $U / U *$ $=12.939)$ 

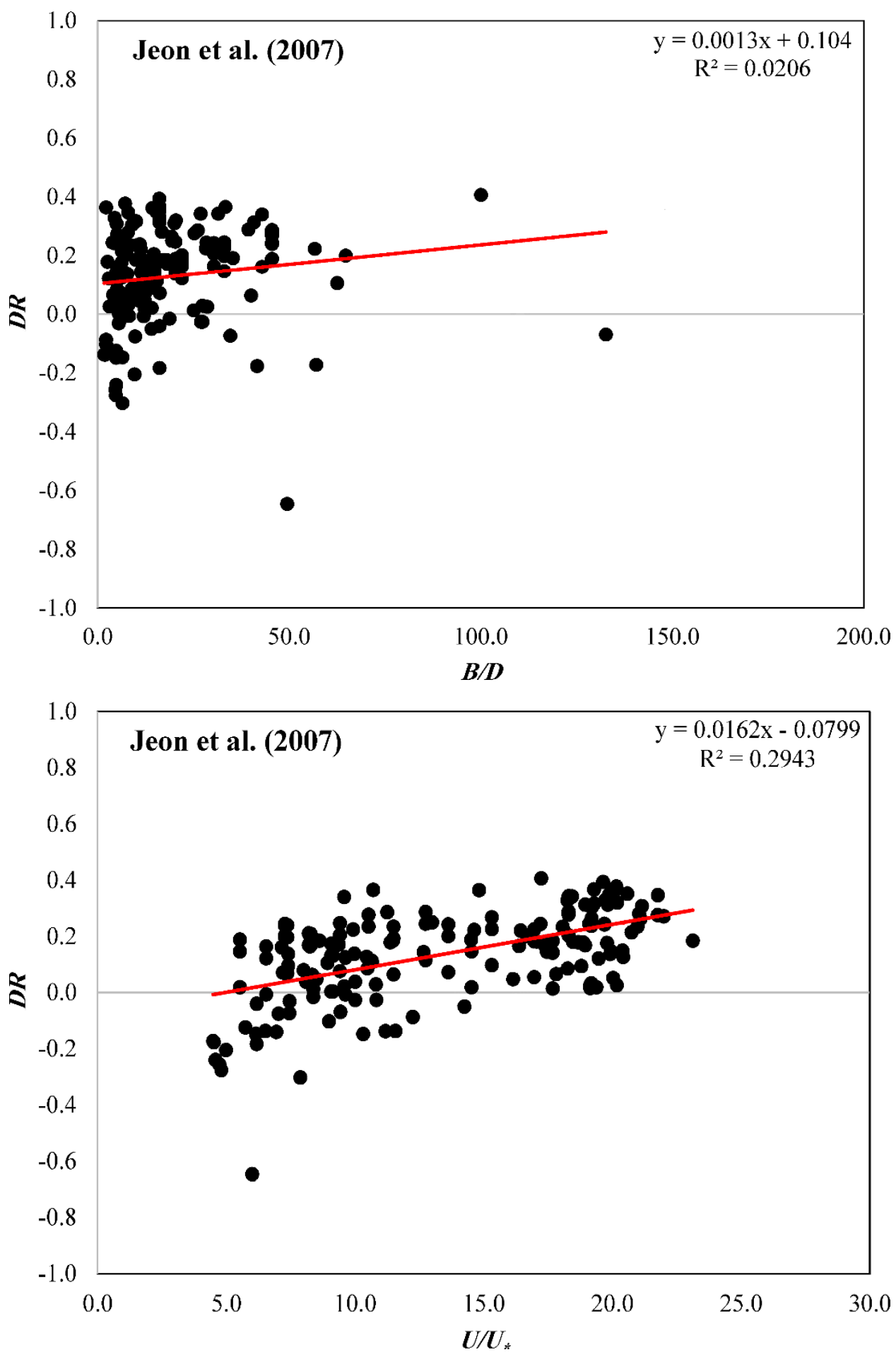

(a) 

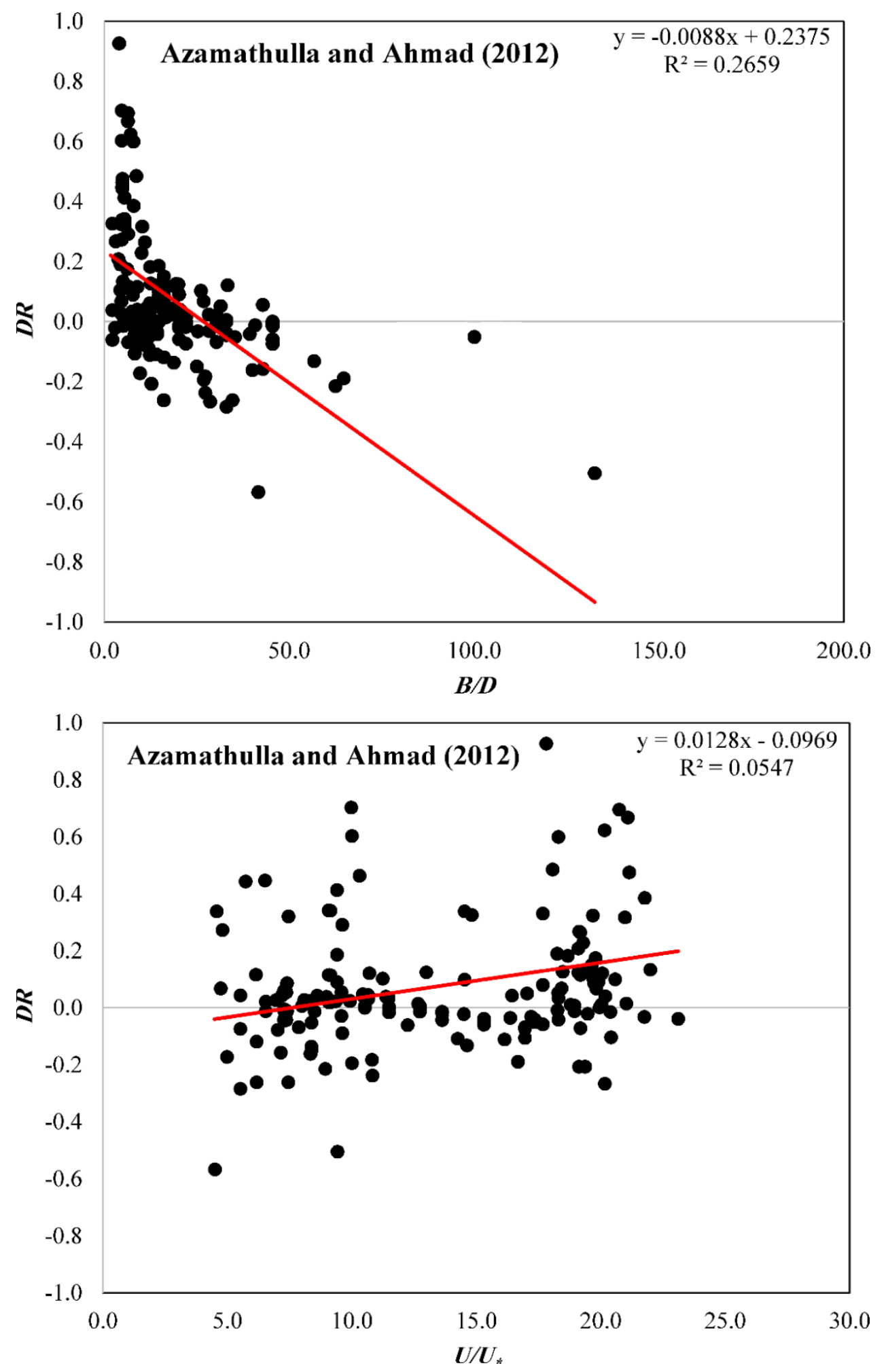

(b) 

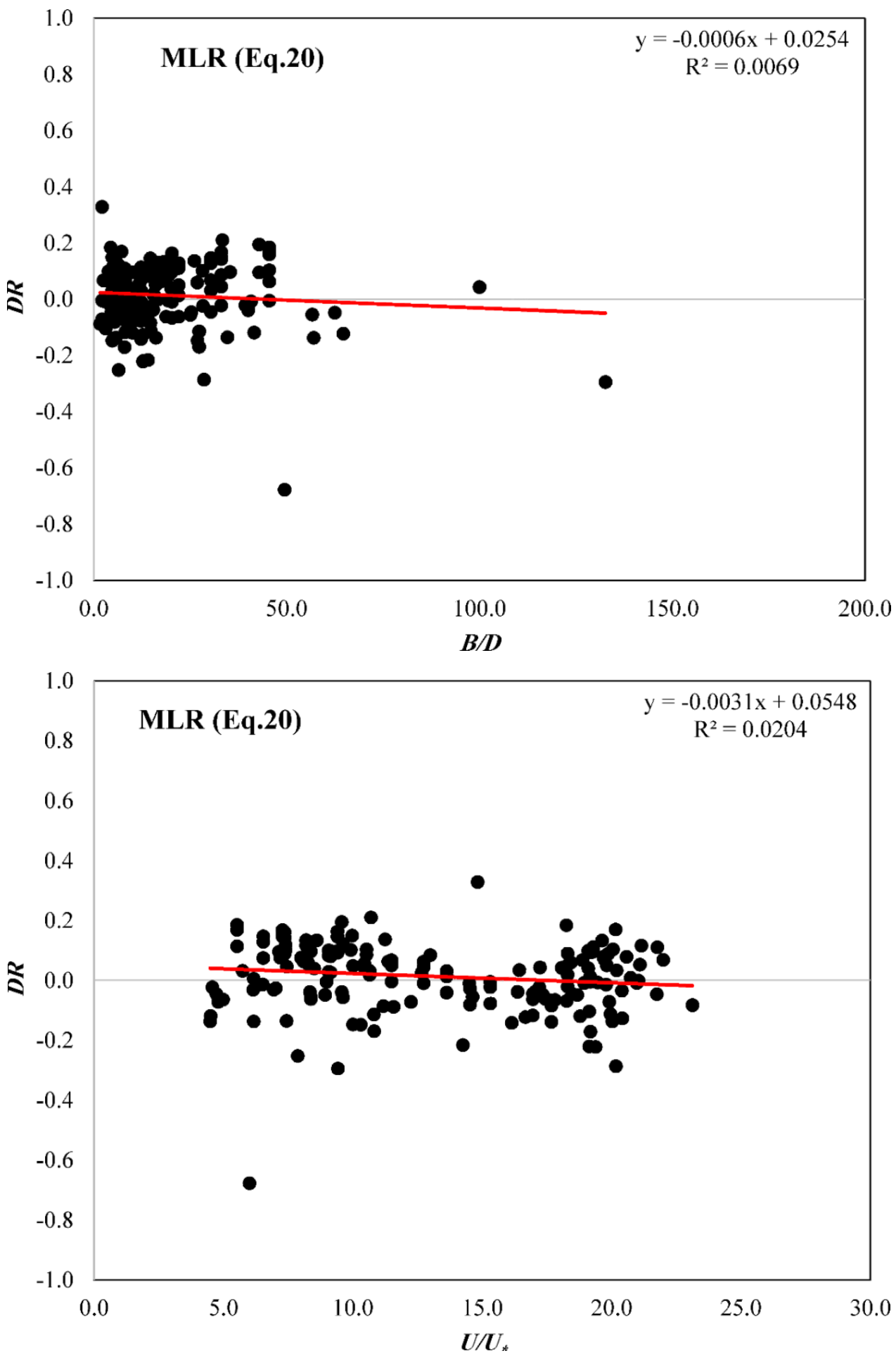

(c) 


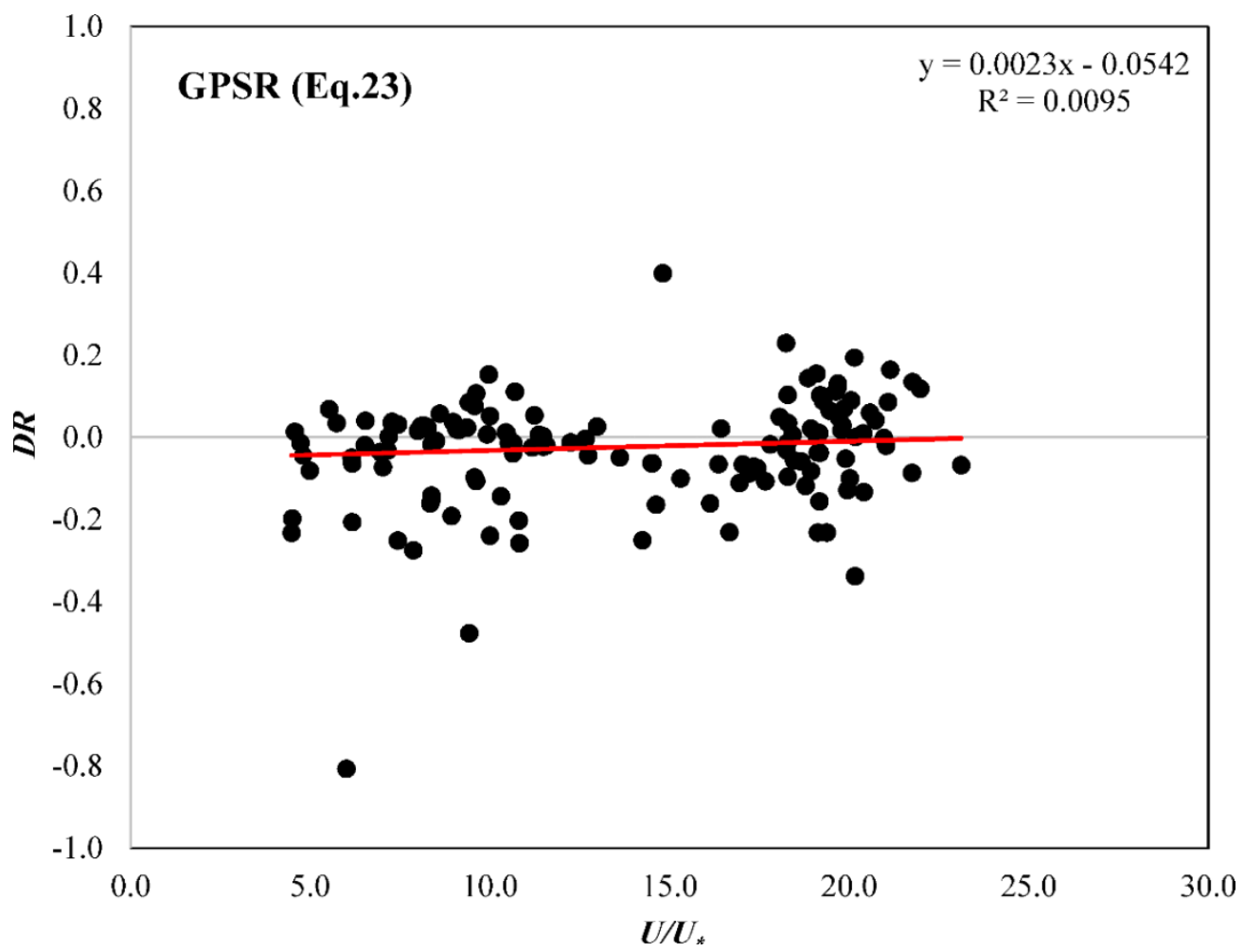

(d)

Fig. 1. Variation of $D R$ against $B / D$ and $U / U *$ for (a) Jeon et al. (2007), (b) Azamathulla and Ahmad (2012), (c) MLR and (d) GPSR 


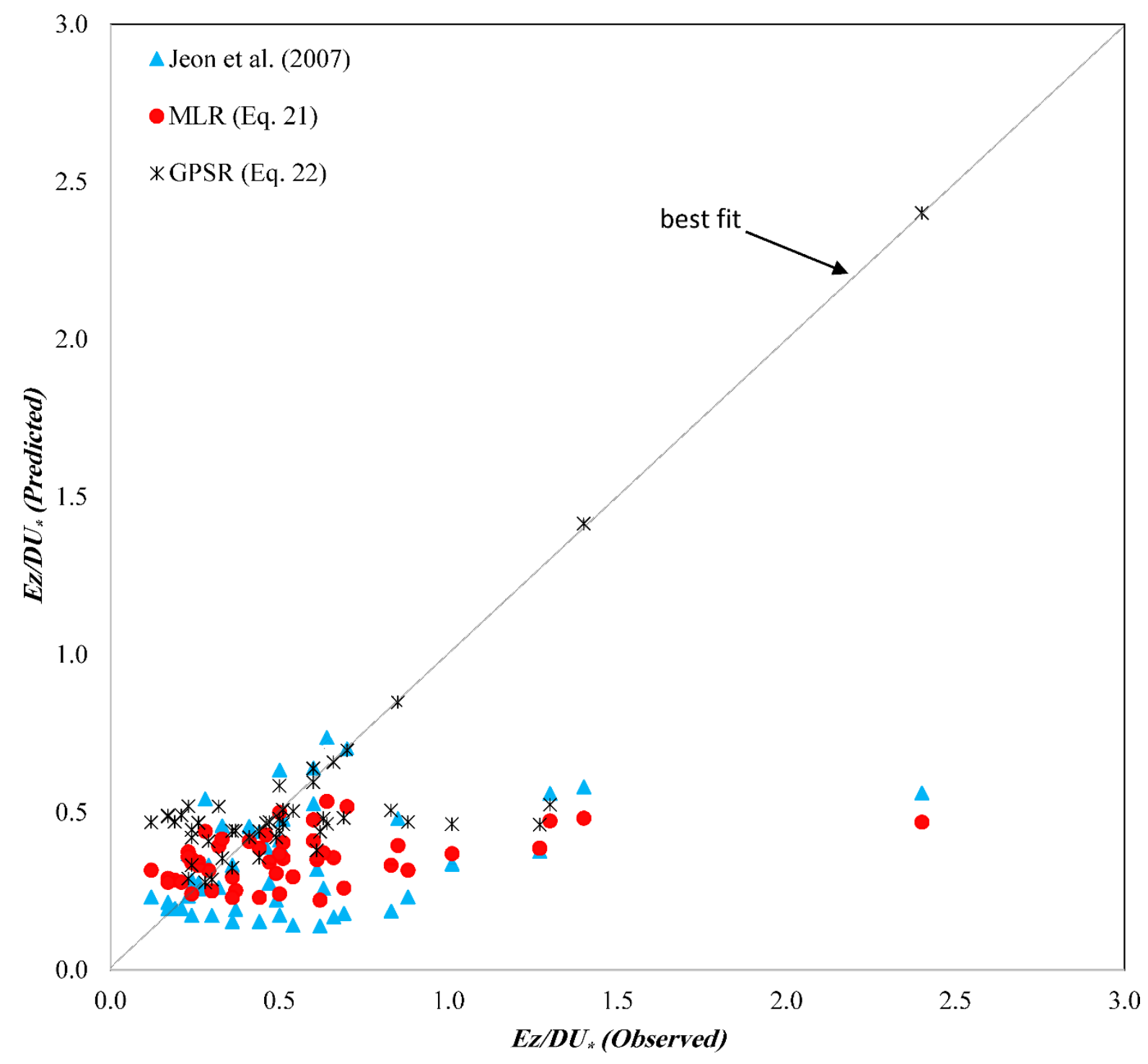

Fig. 2. Comparison of observed and predicted $E_{z} / D U *$ using Jeon et al. (2007), MLR and GPSR models 


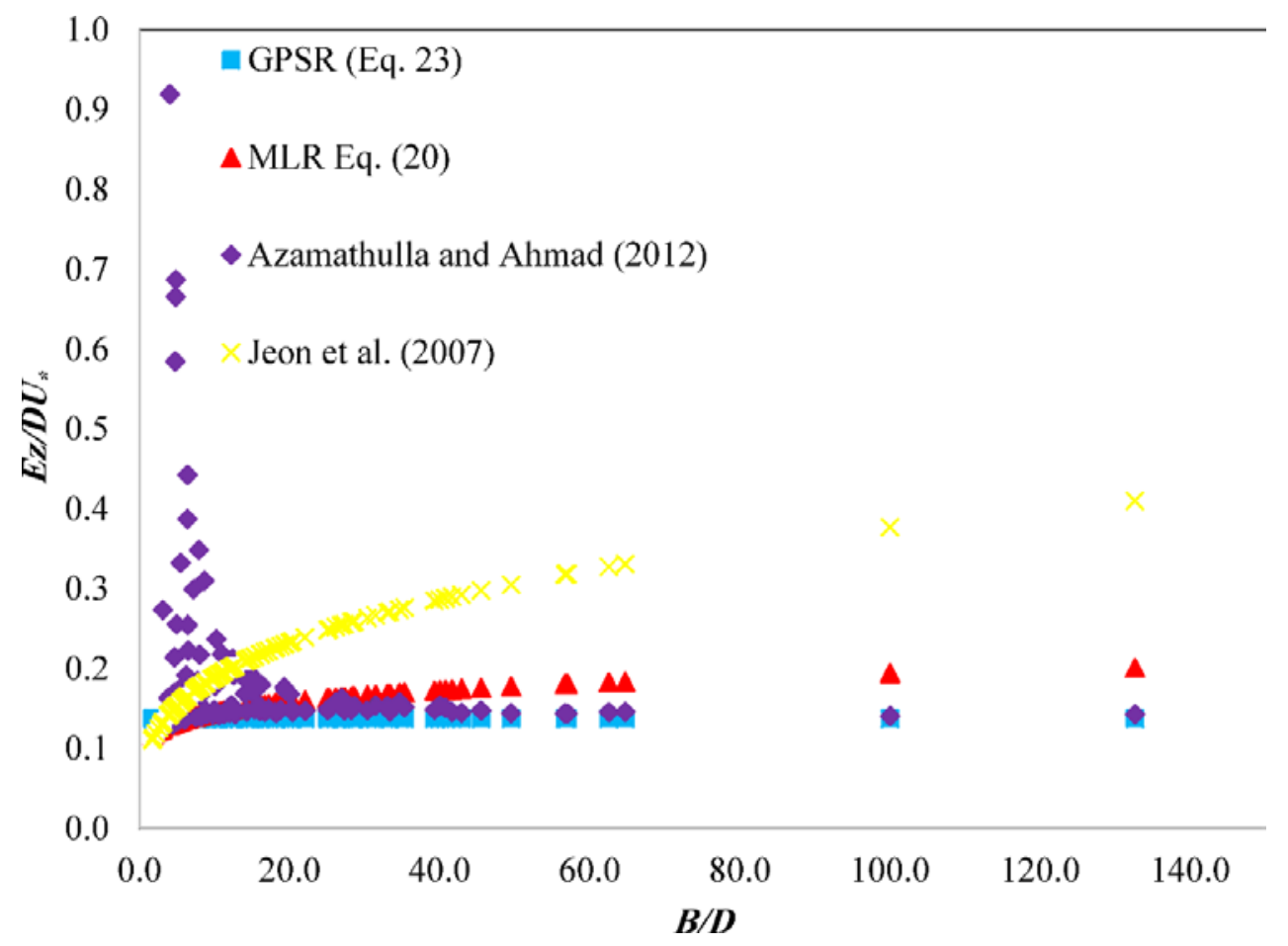

(a)

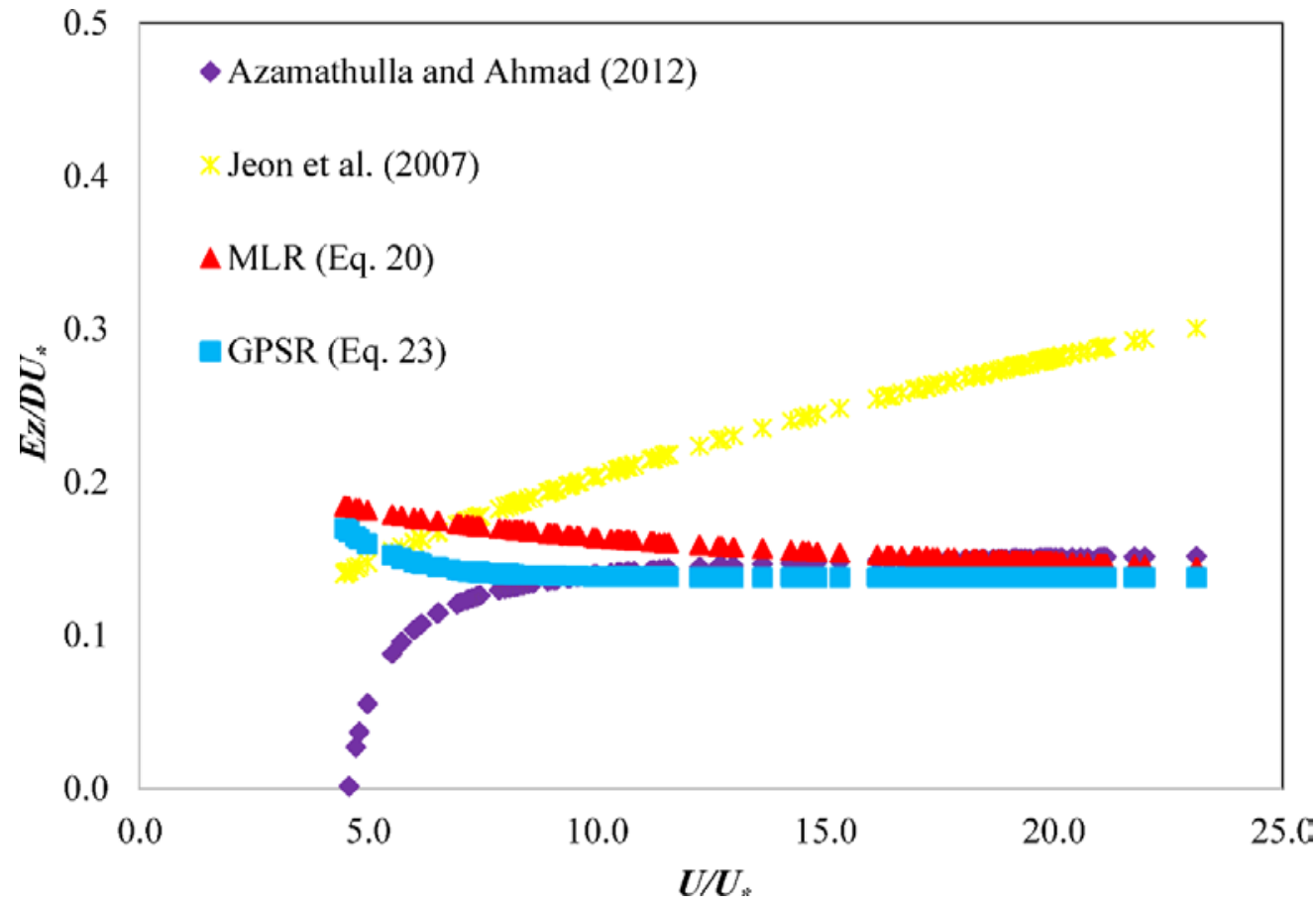

(b)

Fig. 3. $E z / D U_{*}$ versus (a) $B / D$ and (b) $U / U_{*}$ for straight streams models $\left(B / D=19.067\right.$ and $U / U_{*}$ $=12.939)$ 